\title{
Uncertainty Analysis in the Notch Impact Test, for Materials with Different Energy Levels
}

\author{
Bulent Aydemir \\ Tubitak National Metrology Institute (TUBITAK UME) Force Laboratory, \\ Gebze-Kocaeli, Turkey, bulent.aydemir@tubitak.gov.tr
}

\begin{abstract}
The notch impact test, that is used to determine the amount of absorbed energy consumed, for breaking materials, under dynamic forces, is among the various important material mechanical tests. The quality and reliability of the results obtained, as a result of the test, are of great value for laboratories. In this study, the parameters affecting the measurement quality in the notch impact test, are expressed as uncertainty values. Charpy notch impact test was performed for materials with different absorbed energy levels in the laboratory. The uncertainty values were calculated, according to the ISO 148-1 standard, from the data obtained. Then, the uncertainty values were analyzed and their effects on the uncertainty calculation were determined. Herein, it was concluded that the parameters should be determined and analyzed correctly, in order to minimize measurement uncertainties.
\end{abstract}

Keywords: Charpy test; uncertainty calculation; notch impact test; ISO 148-1

\section{Introduction}

Among the mechanical characterization tests, the Charpy notch impact test is used to determine the mechanical properties of materials that work under conditions that can cause brittle fracture. The aim of the impact test in general is to determine the amount of energy and characterizing the fracture behavior of the materials under dynamic forces. This test is a simple, low-cost and reliable test that is mandatory for pipeline manufacturers, bridge construction industry and pressure vessel manufacturers. The impact test can be applied to most materials, but this paper only deals with metallic materials. Charpy notch impact test has been used extensively in mechanical testing of steel products, in research, and in purchase specifications for over four decades [1-3]. Today, there are two standards for general use of Charpy test for metallic materials. One of these standards is "Metallic Materials - Charpy Pendulum Impact Test" ISO 148-1:2016, the other is "Standard Test Methods for Notched Bar Impact Testing of Metallic Materials" ASTM E23:2018. The Charpy test is used to determine the amount of energy 
absorbed during fracture. The Charpy test is most commonly used to evaluate the properties of steels, based on characterizing fracture behavior or impact toughness and is therefore, often used in quality control applications, where it is a fast and economical test. A Charpy impact test measurement setup is given in Figure 1 [4]. It is used more as a comparative test rather than a definitive test. Some of the advantages of Charpy test include;

- This test is relatively easy to perform

- It is used to evaluate the toughness properties of materials

- It is useful for evaluating new products

- It provides fast measurement results

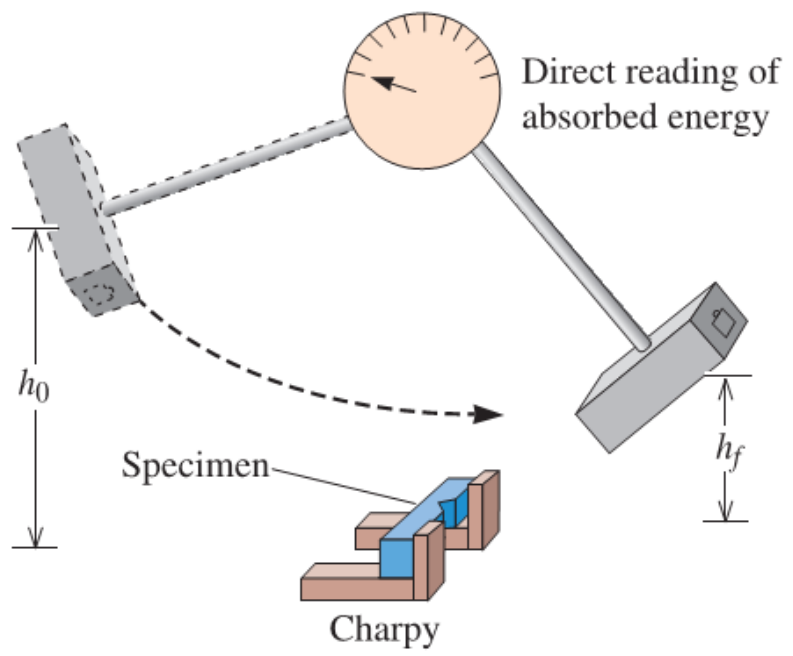

Figure 1

Principle of Charpy notch impact test machine [4]

When reporting the result of a test and analysis measurement, some quantitative indicators of the quality of the result should be given. This way, those who use it can evaluate the reliability of the test. Without such an indicator, measurement results are very difficult to compare among themselves or with reference values given in a specification or standard. Measurement uncertainty, which indicates the quality of a measurement result, is an easily applied, easily understood and generally accepted approach [5-8].

Laboratories operating under ISO/IEC 17025 accreditation and related systems are accordingly required to evaluate measurement uncertainty for test results. Taking into account all uncertainty components, which are of importance in the given situation, is obligatory, when reporting the measurement uncertainty. 
Requirements for laboratories that want to be accredited in the notch impact test "General requirements for the competence of testing laboratories" ISO / IEC 17025:2017 standard has been defined. It includes the evaluation, approval and subsequent inspection of the technical competence of the laboratory according to the necessary criteria by an internationally recognized and authorized organization in order to ensure that the tests and analyzes carried out with ISO / IEC 17025 accreditation can provide confidence. Measurement uncertainty calculation is a desirable requirement.

As it is known, the indicator of measurement quality and the reliability of measurement results is the uncertainty value. For each device calibrated, an uncertainty value for that device is defined in its certificate. According to the accredited test report, it is necessary an uncertainty value must be calculated within the values obtained as a result of the test.

In this study, the uncertainty calculation, definitions, calculation formulas and sample numerical calculations for the notch impact test results are given in detail in sections. Then, the Charpy notch impact test was performed for materials with different absorbed energy levels in the laboratory. Uncertainty values were calculated according to the relevant standard for measurement data. Then, the uncertainty values were analyzed and their effects on the uncertainty calculation were determined. The aim of the study is to reveal what needs to be done in order to obtain a lower uncertainty value for notch impact testing.

\section{Uncertainty Calculation}

Global comparability is based on international comparisons of Charpy reference machines and approved values of certified reference test materials produced by national or international bodies using reference machines. The traceability chain begins internationally with the definition of the absorbed energy measured in the procedures described in the ISO 148-1 standard. Calibration laboratories use certified reference test material to validate reference machines. Besides, at the user level, Charpy test laboratories can verify their pendulum with reference test material to obtain reliable absorbed energy values. When research is conducted within the scope of the measurement uncertainty of the energy value that is absorbed by the specimen during testing, it is seen that the uncertainty analysis and effective factors are detailed in the ISO 148-1 standard. The 2018 version of the ASTM E23 standard does not include an uncertainty approach for the Charpy impact test. In this section, uncertainty calculations and effective factors specified in ISO 148-1, are explained in detail. 


\subsection{Factors Contributing to Uncertainty}

Effective factors that contribute to measurement uncertainty in Charpy impact test are listed below:

a) Machine bias obtained from indirect verification

b) Homogeneity of test material and machine repeatability

c) Test temperature

Measurement equation for average absorbed energy $(K V)$ is defined as formula 1:

$$
K V=\bar{x}-B v-T x
$$

Descriptions of expressions used in formula 1 are as follows:

$\bar{x}$ is observed averaged absorbed energy of $\mathrm{n}$ test specimens

$B v$ is machine bias based on indirect verification

$T x$ is bias due to temperature

\subsection{Machine Bias}

Machine bias is one of the effective factors that contribute to measurement uncertainty in Charpy impact test, so it is determined by indirect verification which is defined in ISO 148-2:2016 standard, as given in formula 2 below:

$$
B v=K V_{v}-K V_{R}
$$

Descriptions of expressions in formula 2 are as follows:

$K V_{V}$ is mean value of reference test specimens fractured during indirect verification

$K V_{R}$ is certified value of reference test material

The uncertainty value of device deviation is calculated using the formulas given in ISO 148-2.

$$
u(B v)=\sqrt{u_{v}^{2}(x)+u_{R M}^{2}}
$$

$u(B V)$ is standard uncertainty of machine bias

$u_{v}(x)$ is standard uncertainty of indirect validation results

$u_{R M}$ is standard certificate uncertainty of reference test samples

$$
u_{v}=\sqrt{u^{2}(B v)+B v^{2}}
$$


$B v$ is machine bias based on indirect verification,

$u_{V}$ is standard uncertainty of indirect verification results

\subsection{Machine Repeatability and Material Homogeneity}

Uncertainty of machine repeatability $u(\bar{x})$ is determined by using formula 5 .

$u(\bar{x})=\frac{s_{X}}{\sqrt{n}}$

$s_{X}=\sqrt{\frac{1}{n-1} \sum_{i=1}^{n}\left(x_{i}-\bar{x}\right)^{2}}$

$n$ is number of tested samples, $x_{i}$ is absorbed energy value of the tested sample, $\bar{x}$ is the average absorbed energy value of $\mathrm{n}$ samples, $s_{X}$ is the standard deviation of absorbed energy values obtained on $n$ test samples. The value $S_{X}$ is caused by two factors, one is machine repeatability and the other is specimen material homogeneity.

\subsection{Temperature Bias}

Effect of temperature bias $T x$ on absorbed energy is extremely material dependent. If steel is tested in brittle-to-ductile transition region, small changes in temperature can correspond to large differences in absorbed energy.

\subsection{Machine Resolution}

Effect of machine resolution is in most cases negligible in comparison with other uncertainty contributions. An exception is the case where machine resolution is large and measured energy is low. In that case, corresponding uncertainty contribution of machine resolution $u(r)$ is calculated using formula 7 :

$u(r)=\frac{r}{\sqrt{3}}$

$r$ is machine resolution in formula (7) 


\subsection{Combined and Expanded Uncertainty}

In order to calculate combined uncertainty value of Charpy impact test measurement $u(K V)$, factors contributing to test uncertainty should be combined using formula (8) below.

$$
u(K V)=\sqrt{u^{2}(\bar{x})+u_{V}^{2}+u^{2}(r)}
$$

Finally, expanded uncertainty value of Charpy impact test measurement $U(K V)$ is calculated using formula (9) below.

$U(K V)=k \cdot u(K V)=t_{95}\left(v_{\overline{K V}}\right) \cdot u(K V)$

$t_{95}\left(v_{\overline{K V}}\right)$ is fraction value having a $95 \%$ confidence level corresponding to measurement degrees of freedom.

For expanded uncertainty calculation, first of all, measurement degree of freedom value $v_{\overline{K V}}$ should be calculated using formula (10) below.

$v_{\overline{K V}}=\frac{u^{4}(\overline{K V})}{\frac{u^{4}(\bar{x})}{v_{\bar{x}}}+\frac{u_{v}^{4}}{v_{v}}}$

$v_{v}$ is degrees of freedom corresponding with $u_{v}, v_{\bar{x}}$ is degrees of freedom corresponding with $u(\bar{x})$.

Then the fraction value corresponding to the degrees of freedom calculated by the formula (10) and having a 95\% confidence level is determined. Table 1 has given $t p(v)$ fraction values corresponding to measurement degrees of freedom.

Table 1

Determination of $t p(v)$ value according to degree of freedom

\begin{tabular}{|c|c|}
\hline Degrees of freedom, $v$ & $t p(v)$ for fraction $P=95 \%$ \\
\hline 1 & 12.71 \\
\hline 2 & 4.30 \\
\hline 3 & 3.18 \\
\hline$\ldots$ & $\ldots$ \\
\hline 7 & 2.36 \\
\hline 8 & 2.31 \\
\hline 9 & 2.26 \\
\hline 10 & 2.23 \\
\hline$\ldots$ & $\ldots$ \\
\hline$\ldots$ & $\ldots$ \\
\hline
\end{tabular}




\begin{tabular}{|c|c|}
\hline 100 & 1.98 \\
\hline$\infty$ & 1.96 \\
\hline
\end{tabular}

\section{Test Methods and Analysis of Uncertainty}

The Charpy notch impact tests were performed by using a machine $300 \mathrm{~J}$ capacity. The impact testing machine has a certification, which was verified both with the direct and indirect verification according to ISO 148-2.

Charpy impact test specimens with different energy levels prepared concerning to ISO 148-1 standard. The dimensions of the all samples were checked against the criteria specified in ISO 148-1: length $(55.00 \pm 0.06) \mathrm{mm}$, height $(10.00 \pm 0.11)$ $\mathrm{mm}$, width $(10.00 \pm 0.075) \mathrm{mm}$, notch angle $(45 \pm 2)^{\circ}$, height remaining at notch root $(8.00 \pm 0.075) \mathrm{mm}$, radius at notch root $(0.25 \pm 0.025) \mathrm{mm}$, distance between the plane of symmetry of the notch and the longitudinal axis of the test piece $(27.50 \pm 0.42) \mathrm{mm}$. The surface roughness of the test samples is better than $5 \mu \mathrm{m}$ and meets all other requirements.

The measurement results of Charpy impact test specimens with different energy levels prepared according to ISO 148-1 standard are presented in Table 2 below. In table, measurement results of five Charpy impact test specimens with six group (E1, E2, E3, E4, E5, E6) different energy levels, their mean value, their standard deviation value and uncertainty value have been presented as Joule unit respectively.

Table 2

Measurement results of test specimens

\begin{tabular}{|c|c|c|c|c|c|c|c|c|}
\hline \multirow{2}{*}{ Code } & \multicolumn{5}{|c|}{ Measurement Results, J } & $\begin{array}{c}\text { Mean } \\
\text { Value, } x\end{array}$ & $\begin{array}{c}\text { Standard } \\
\text { Deviation, } \\
s_{x}\end{array}$ & $\begin{array}{c}\text { Uncertainty, } \\
u(x)\end{array}$ \\
\cline { 2 - 9 } & 1 & 2 & 3 & 4 & 5 & $\mathrm{~J}$ & $\mathrm{~J}$ & $\mathrm{~J}$ \\
\hline E1 & 21.2 & 21.7 & 22.7 & 21.8 & 22.3 & 21.94 & 0.57 & $\mathbf{0 . 2 6}$ \\
\hline E2 & 30.4 & 37.2 & 29.5 & 31.6 & 31.8 & 32.10 & 3.00 & $\mathbf{1 . 3 4}$ \\
\hline E3 & 67.2 & 65.6 & 68.4 & 63.5 & 68.8 & 66.70 & 2.20 & $\mathbf{0 . 9 8}$ \\
\hline E4 & 105.5 & 110.5 & 111.9 & 105.6 & 112.1 & 109.12 & 3.32 & $\mathbf{1 . 4 4}$ \\
\hline E5 & 124.3 & 119.8 & 122.8 & 123.9 & 114.5 & 121.06 & 4.07 & $\mathbf{1 . 8 2}$ \\
\hline E6 & 171.5 & 177.1 & 168.5 & 167.7 & 177.5 & 172.46 & 4.64 & $\mathbf{2 . 0 8}$ \\
\hline
\end{tabular}

Calculations of statistical expressions located in Table 2 are explained in detail below. First, the arithmetic average of measurement results should be calculated for every code. The standard deviation of measurement results in Table 2 are calculated using following formula (6). Then the standard uncertainty of absorbed energy of measurement results should be calculated using formula 5 . 
When measurement results in Table 2 are examined, resolution of Charpy impact test machine is determined to be 0.1 Joule. Thus, uncertainty component of machine resolution is calculated using following formula (7).

$u(r)=\frac{r}{\sqrt{3}}=\frac{0.1}{\sqrt{3}}$

$u(r)=0.06 \mathrm{~J}$

In order to determine standard uncertainty of indirect verification measurement, reference samples with known certificate values should be used, which are given in Table 3 below.

Table 3

Certificate value of reference test specimen

\begin{tabular}{|c|c|c|c|c|c|}
\hline Code & $\begin{array}{c}\text { Certificate } \\
\text { Number }\end{array}$ & Traceability & $\begin{array}{c}\text { Certificate } \\
\text { value, } \mathrm{KV}_{R}\end{array}$ & $\begin{array}{c}\text { Degree of } \\
\text { Freedom }\end{array}$ & $\begin{array}{c}\text { Certificate } \\
\text { Uncertainty } \\
(\mathrm{k}=2), U_{R M}\end{array}$ \\
\hline- & - & - & $\mathrm{J}$ & VV RM & $\mathrm{J}$ \\
\hline $\mathrm{R} 1$ & $\mathrm{XXX}$ & $\mathrm{XXX}$ & 123.8 & 7 & 3.4 \\
\hline
\end{tabular}

Certified reference material has been fractured using Charpy impact testing machine with the method specified in ISO 148-2 standard. Measurement results are also given in Table 4 below.

Table 4

Measurement results of reference test sample

\begin{tabular}{|c|c|c|c|c|c|c|c|}
\hline \multicolumn{4}{|c|}{ Measurement Results, J } & $\begin{array}{c}\text { Mean } \\
\text { Value, } \\
K V_{V}\end{array}$ & $\begin{array}{c}\text { Standard } \\
\text { Deviation, } s_{x}\end{array}$ & $\begin{array}{c}\text { Uncertainty, } \\
u_{v}(x)\end{array}$ \\
\hline 1 & 2 & 3 & 4 & 5 & $\mathrm{~J}$ & $\mathrm{~J}$ & $\mathrm{~J}$ \\
\hline 123.1 & 116.1 & 112.8 & 123.6 & 121.3 & 119.4 & 4.7 & 2.1 \\
\hline
\end{tabular}

Calculations of statistical expressions located in Table 4 are explained in detail below. Arithmetic average, standard deviation and uncertainty values of measurement results should be calculated as similar Table 2 .

Using to formula (2), the estimate of Charpy impact test machine bias is calculated as follows,

$$
B v=K V_{v}-K V_{R}=119.4-123.8
$$

$B v=-4.4 \mathrm{~J}$

Also, standard uncertainty of pendulum impact testing machine bias is determined by using formula (3) specified in ISO 148-2 standard below. 
$u(B v)=\sqrt{u_{v}^{2}(x)+u_{R M}^{2}}=\sqrt{2.1^{2}+(3.4 / 2)^{2}}$

$u(B v)=2.71 \mathrm{~J}$

As a general rule, bias should be corrected, however due to wear of anvils and hammer parts; it is difficult to obtain a perfectly stable bias value throughout period between two indirect verifications. This is why measured bias value is often considered an uncertainty contribution, to be combined with its own uncertainty to obtain uncertainty of indirect verification result.

$u_{v}=\sqrt{u^{2}(B v)+B_{V}^{2}}=\sqrt{2.71^{2}+(-4.4)^{2}}$

$u_{v}=5.19 \mathrm{~J}$

In order to calculate combined uncertainty value of Charpy impact test measurement, factors contributing to uncertainty should be combined as defined in ISO 148-1 standard below,

$$
u_{E 1, E 2, E 3, E 4, E 5, E 6}(K V)=\sqrt{u^{2}(\bar{x})+u_{V}^{2}+u^{2}(r)}=\sqrt{u_{E 1, E 2, E 3, E 4, E 5, E 6}^{2}(\bar{x})+5.19^{2}+0.06^{2}}
$$

Measurement degree of freedom value is calculated using formula 10 below.

$$
v_{\overline{K V}}=\frac{u^{4}(\overline{K V})}{\frac{u^{4}(\bar{x})}{v_{\bar{x}}}+\frac{u_{v}^{4}}{v_{v}}}=\frac{u_{E 1, E 2, E 3, E 4, E 5, E 6}^{4}(\overline{K V})}{\frac{u_{E 1, E 2, E 3, E 4, E 5, E 6}^{4}(\bar{x})}{5-1}+\frac{5.19^{4}}{7}}
$$

Concerning to Table $1, t_{p}$ values are determined as Table 5. Finally, expanded uncertainty of $U(K V)$ is determined using formula 9 as follows.

$$
U(K V)=k \cdot u(K V)=t_{95}\left(v_{\overline{K V}}\right) \cdot u(K V)
$$

All uncertainty parameters calculated in numerical are summarized in Table 5 below.

Table 5

For different energy levels, calculated measurement uncertainties and values of related parameters

\begin{tabular}{|c|c|c|c|c|c|c|c|c|c|c|}
\hline \multirow{2}{*}{ Code } & $\mathrm{KV}_{R}$ & $U_{R M}$ & $B v$ & $u(B v)$ & $u_{v}$ & $u(r)$ & $u(x)$ & $u(K V)$ & $V_{K V}$ & $U(K V)$ \\
\cline { 2 - 12 } & $\mathrm{J}$ & $\mathrm{J}$ & $\mathrm{J}$ & $\mathrm{J}$ & $\mathrm{J}$ & $\mathrm{J}$ & $\mathrm{J}$ & $\mathrm{J}$ & - & $\mathrm{J}$ \\
\hline E1 & 123.8 & 3.4 & -4.4 & 2.71 & $\mathbf{5 . 1 9}$ & $\mathbf{0 . 0 6}$ & $\mathbf{0 . 2 6}$ & 5.19 & 7.0 & $\mathbf{1 2 . 0 0}$ \\
\hline E2 & 123.8 & 3.4 & -4.4 & 2.71 & $\mathbf{5 . 1 9}$ & $\mathbf{0 . 0 6}$ & $\mathbf{1 . 3 4}$ & 5.36 & 7.9 & $\mathbf{1 2 . 6 4}$ \\
\hline E3 & 123.8 & 3.4 & -4.4 & 2.71 & $\mathbf{5 . 1 9}$ & $\mathbf{0 . 0 6}$ & $\mathbf{0 . 9 8}$ & 5.28 & 7.5 & $\mathbf{1 2 . 3 3}$ \\
\hline E4 & 123.8 & 3.4 & -4.4 & 2.71 & $\mathbf{5 . 1 9}$ & $\mathbf{0 . 0 6}$ & $\mathbf{1 . 4 4}$ & 5.38 & 8.0 & $\mathbf{1 2 . 4 4}$ \\
\hline E5 & 123.8 & 3.4 & -4.4 & 2.71 & $\mathbf{5 . 1 9}$ & $\mathbf{0 . 0 6}$ & $\mathbf{1 . 8 2}$ & 5.50 & 8.6 & $\mathbf{1 2 . 5 3}$ \\
\hline E6 & 123.8 & 3.4 & -4.4 & 2.71 & $\mathbf{5 . 1 9}$ & $\mathbf{0 . 0 6}$ & $\mathbf{2 . 0 8}$ & 5.59 & 9.0 & $\mathbf{1 2 . 6 3}$ \\
\hline
\end{tabular}




\section{Discussion}

The following equation was used to calculate the expanded measurement uncertainty value based on the Charpy notch impact test measurements.

$$
U(K V)=k \cdot \sqrt{u^{2}(\bar{x})+u_{V}^{2}+u^{2}(r)}
$$

When the measurement uncertainty values given in Table 5 are analyzed, since all samples are tested on the same calibrated impact device, $u_{v}$ and $u(r)$ values are taken into account as the same value. It is only $u(x)$ whose value changes in the measurement uncertainty calculation. In order to better understand the parameters that affect this uncertainty calculation, the effect percentages are given in Figure (2). It is the parameter that has the greatest impact in uncertainty calculation was $u_{v}$ with an average value of $80 \%$ in Figure (2). The average effect of the $u(x)$ value is $19 \%$ and the average effect of the $u(r)$ value is $1 \%$.

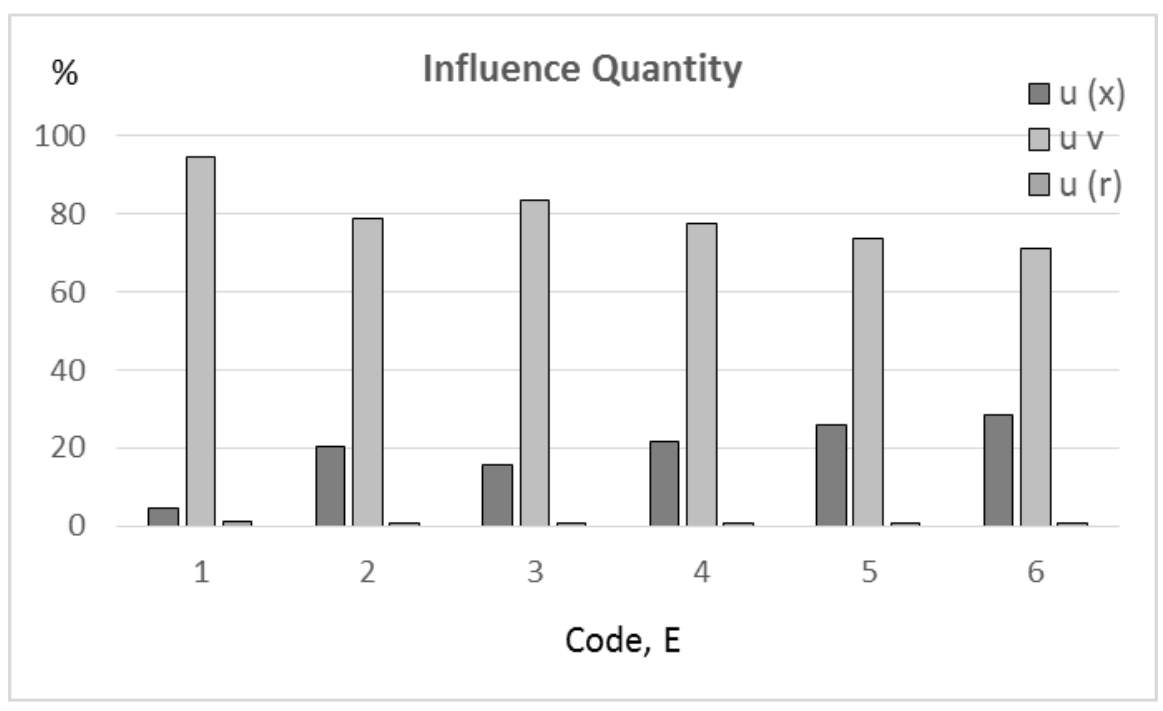

Figure 2

Influence quantity in the uncertainty calculation for code E1, E2, E3, E4, E5, E6 samples

For a more detailed analysis of the $u_{v}$ parameter, which is the greatest impact in the uncertainty calculation, the calculation of the $u_{v}$ parameter should be examined. The following equation is used to calculate the measurement uncertainty of indirect verification from the Charpy notch impact test machine.

$$
u_{v}=\sqrt{u^{2}(B v)+B v^{2}}=\sqrt{u_{v}^{2}(x)+u_{R M}^{2}+B v^{2}}
$$

As can be seen from the equation, three parameters are included in the uncertainty calculation. To see the changes in these parameters, the Charpy notch impact 
tester used in the tests was calibrated using reference test pieces with different energy levels. The values and results of the reference test pieces belonging to different energy levels used in the calibration of the notched notch impact test machine are given in Table 6. Reference test pieces (CRM) are shown with the codes R2, R3, R4. Using these reference pieces, the measurements were taken on the same Charpy impact testing machine and the $u_{v}(x)$ and $B v$ values were determined.

The effect percentages of the parameters that affect the standard uncertainty of the indirect validation results in Table 6 are given in Figure (3). $U_{R M}$ was the greatest impact parameter in the uncertainty between $35 \%$ and $55 \%$ in the calculation of indirect verification results. Similar results were obtained in the study by NIST [9] [10]. In figure (3), the average effect of the $B v$ value is $34 \%$ and the average effect of the $u_{v}(x)$ value is $20 \%$. As a result, the parameters that have a major impact on the uncertainty calculation are determined as the uncertainty value of the $U_{R M}$ reference test pieces and the deviation error in the $B v$ reference test sample values.

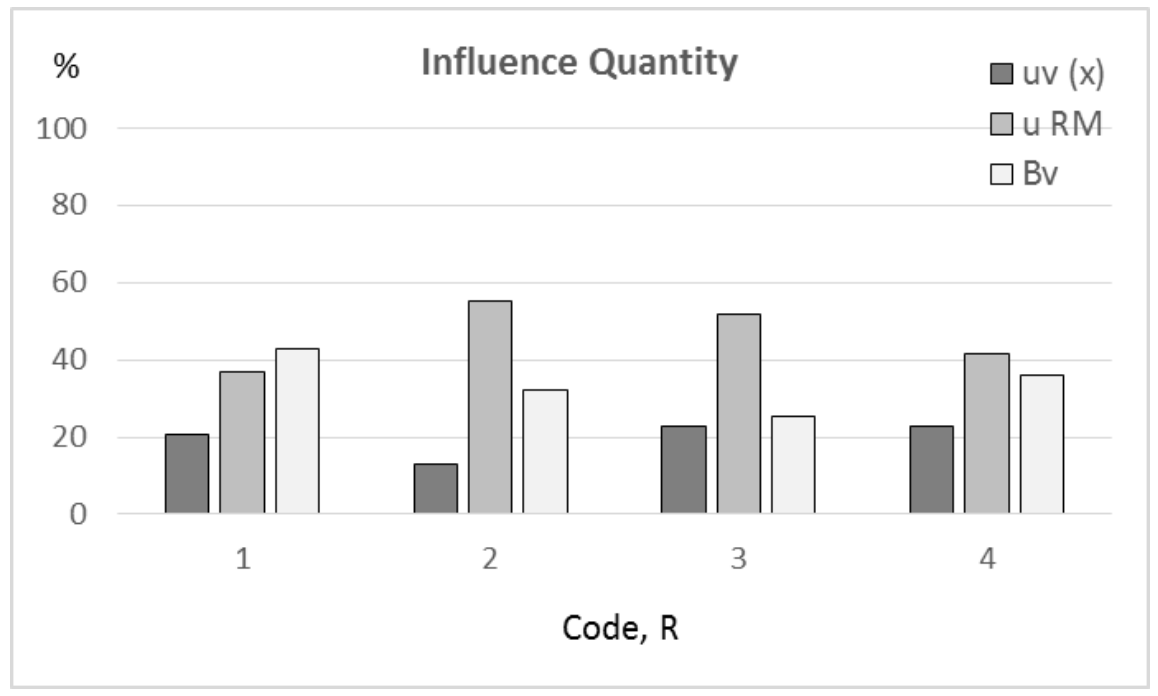

Figure 3

Influence quantity in the uncertainty calculation for code R1, R2, R3, R4 of CRMs

As a result, the parameters that are the impact in the uncertainty calculation in the notch impact testing machine and the percentages of their effects are given. There are different references for uncertainty calculations for notch impact testing [1114]. However, there is no analysis study on uncertainty calculation in this area. The laboratory that wants to reduce the uncertainty value of the notch impact test can solve its problem by examining the calculations given in this study. In this study, the uncertainty calculation in the notch impact test was analyzed and the greatest impact parameters for the uncertainty calculation related to the subject 
were presented to accredited laboratories and institutions using the Charpy notch impact test.

Table 6

For different energy levels of reference Charpy test pieces' materials, certificated and calculated uncertainties and values of related parameters

\begin{tabular}{|c|c|c|c|c|c|c|}
\hline Code & $\begin{array}{c}\text { Certificate } \\
\text { value, } K V_{R}\end{array}$ & $\begin{array}{c}\text { Degree of } \\
\text { Freedom }\end{array}$ & $k$ & $\begin{array}{c}\text { Certificate } \\
\text { Uncertainty, } \\
U_{R M}\end{array}$ & $\begin{array}{c}\text { Uncertainty, } \\
u_{v}(x)\end{array}$ & $B v$ \\
\hline- & $\mathrm{J}$ & $V_{V R M}$ & $t_{p}$ & $\mathrm{~J}$ & $\mathrm{~J}$ & $\mathrm{~J}$ \\
\hline R2 & 216.3 & 35 & 2.03 & $\mathbf{8 . 6}$ & $\mathbf{2 . 0}$ & $\mathbf{5 . 0}$ \\
\hline R3 & 195.6 & 54 & 2.01 & $\mathbf{7 . 3}$ & $\mathbf{3 . 2}$ & $\mathbf{3 . 6}$ \\
\hline R4 & 21.9 & 35 & 2.03 & $\mathbf{2 . 2}$ & $\mathbf{1 . 2}$ & $\mathbf{1 . 9}$ \\
\hline
\end{tabular}

\section{Conclusions}

In this study, the general uncertainty calculation of the Charpy impact test is explained in detail, in accordance with the ISO 148-1 standard. For the absorbed energy value obtained, as a result of the Charpy impact test, the effective factors contributing to the measurement uncertainty calculation are specified. In order to provide a better understanding of the methods and formulas in the uncertainty approach, the uncertainty calculation for different energy level test samples, is presented in this work as well. Two groups of three-parameter uncertainty parameters, that are the impact in uncertainty calculating of the Charpy test are defined. Its effects on uncertainty calculation have been analyzed. In the first group, the parameters included in the uncertainty calculation are $u(x), u_{v}$ and $u(r)$ values. When all samples were tested on the same notch impact test machine, the $u_{v}$ and $u(r)$ values were taken into account as the same value. In the measurement uncertainty calculation, only the $u(x)$ value determined from the scattering of the test results, was taken into account, as the variable value. For the parameters included in the uncertainty calculation in the first group, the $u_{v}$ value was observed to be the greatest impact parameter in the uncertainty calculation, with an average of $80 \%$. In the second group, the parameters of $u_{v}(x), u_{R M}$ and $B v$ values were examined in the uncertainty calculation of the $u_{v}$ value. By using different reference test specimens, measurements were taken using the same Charpy notch impact test machine and $u_{v}(x)$ and $B v$ values were determined. The $u_{R M}$ value was calculated as the greatest impact parameter in the second group uncertainty parameters, with an average of $46 \%$. Then the average effect of the $B v$ value was calculated as $34 \%$.

To develop the uncertainty value, which is an indicator of measurement quality, it is necessary to analyze the uncertainty calculation well. According to the analysis given in this study, in order to improve the notch impact test uncertainty value, it is necessary to reduce the greatest impact parameters. In this study, the uncertainty calculation, in the notch impact test, was examined and effective parameters, to reduce the uncertainty value were presented. To reduce the uncertainty value of 
notch impact testers, $u_{v}$ the greatest impact parameter should be reduced. Examining the calculation of $u_{v}$ the most influential parameter comes from the uncertainty of the reference materials, $u_{R M}$. To reduce this parameter, choose a reference material with a lower uncertainty value. In this way, a reduction in the uncertainty calculation is achieved. Low uncertainty value can be attained by analyzing other effective parameters, similarly, in the uncertainty calculation.

Practically, the measurement uncertainty value, is calculated from the effective parameters that can cause a change in a measurement result. Measurement uncertainty, defines an uncertainty band at a 95\% confidence level according to the uncertainty approach. By lowering the uncertainty value and narrowing the uncertainty band, it allows for results with higher quality and much closer to the average values.

\section{References}

[1] Aydemir, B. "Application of Measurement Uncertainty in Notch Impact Tests", 3nd International Conference on Material Science and Technology in Cappadocia (IMSTEC'18), Nevsehir (17-19/09/2018): 5 p.

[2] Aydemir, B., 2011, Training of calibration of notch impact test machines, G2KV-050, Oct. 2011, TUBITAK UME

[3] Aydemir, B., 2017, Training of uncertainty calculation for material testingG2KV-120, May 2017, ISDEMIR, Iskenderun

[4] Askeland,_ D. R., Wright, W. J.,_2014, The Science and Engineering of Materials, $7^{\text {th }}$ edition, Cengage Learning

[5] Evaluation of measurement data - Guide to the expression of uncertainty in measurement, JCGM 100:2008

[6] EA guidelines on the expression of uncertainty in quantitative testing, EA4/16, G:2003

[7] T. M. Adams, A2LA Guide for Estimation of Measurement Uncertainty in Testing, July 2002, Guidance, G104

[8] Stephanie Bell, A Beginner's Guide to Uncertainty of Measurement, 1999

[9] Lucon, E., McCowan, C., "Impact Testing Yesterday and Today" ASTM Workshop, 13/11/2011, NIST

[10] J. D. Splett H. K. Iyer C.-M. Wang, C. N. McCowan, Special Publication 960-18 NIST Recommended Practice Guide: Computing Uncertainty for Charpy Impact Machine Test Results, 2008

[11] Lont, M. A., "The Determination of Uncertainties in Charpy Impact Testing," Manual of Codes of Practice for the Determination of Uncertainties in Mechanical Tests on Metallic Materials, UNCERT COP 06:2000, 2000 
[12] Takagi, S. and Yamaguchi, Y., "Uncertainty Analyses of Reference Specimens for the Verification of Charpy Impact Test Machines," J. of Material Testing Research Association of Japan, Vol. 48, No. 4, 2003

[13] Splett, J. D. and Wang, C. M., "Uncertainty in Reference Values for the Charpy V-Notch Verification Program," J. Testing and Evaluation, 2002, pp. 362-369

[14] Roebben, G., Lamberty, A. and Pauwels J. "Certification of Charpy VNotch Reference Test Pieces at IRMM" Journal of ASTM International, July/August 2005, Vol. 2, No. 7 